\title{
Comparison of FDTD Hard Source With FDTD Soft Source and Accuracy Assessment in Debye Media
}

\author{
Fumie Costen, Member, IEEE, Jean-Pierre Bérenger, Fellow, IEEE, and Anthony K. Brown, Senior Member, IEEE
}

\begin{abstract}
To radiate electromagnetic energy from a single point of a finite difference time domain (FDTD) grid, there are typically two general classes of electromagnetic wave sources; the soft source which consists of impressing a current, and the hard source which consists of impressing an electric field. The physical meaning of the soft source is well understood and its analytical solution is known, whereas there is no analytical solution for the hard source excitation. Nevertheless, many FDTD works utilize the hard source for its practicality. A novel aspect is that the derivation of a field radiated from the hard source towards the free space is identical to the field radiated from the soft source, provided that a certain relationship holds between the source excitations. This provides us with an analytical solution for the field radiated from the hard source. The assessment of accuracy is then considered for a wide band field radiated from a punctual source into frequency-dependent FDTD Debye media. The quantification of the deviation of the waveform observed in the FDTD space from the analytical solution is demonstrated. The numerical experiments with this quantification show that the waveform observed with the soft source excitation matches the one with the hard source excitation when the minimum wavelength to the spatial discretization ratio is greater than 10. It turns out that the soft source outperforms the hard source when the minimum wavelength relative to the spatial discretization is less than 10 in the case of lossless media. Equivalent accuracy is achievable for both lossless and lossy media even when the minimum wavelength to the spatial discretization ratio is lower than 10 due to the loss tangent which absorbs the spurious frequencies related to the numerical noise.
\end{abstract}

Index Terms-Debye media, finite difference time domain (FDTD), hard source, punctual source excitation, soft source, ultrawideband (UWB).

\section{INTRODUCTION}

$\mathbf{U}$ LTRAWIDEBAND (UWB) system development requires numerical simulations with detailed modelling of objects, reproducing the behavior of physical electromagnetic waves such as waveform distortion in the time domain during propagation in a wide range of media such as a lossy human body. The finite difference time domain (FDTD) method works in the time domain and is capable of explicitly computing macroscopic transient electromagnetic interactions with general three dimensional geometries. However, the original FDTD

Manuscript received February 11, 2008; revised February 16, 2009. First published May 02, 2009; current version published July 09, 2009.

F. Costen and A. K. Brown are with the School of Electrical and Electronic Engineering, The University of Manchester, Manchester M60 1QD, U.K. (e-mail: f.costen@cs.man.ac.uk; a.brown@manchester.ac.uk).

J.-P. Bérenger is with the Centre d'Analyse de Défense, 94114 Arcueil,

France (e-mail: jean-pierre.berenger@dga.defense.gouv.fr).

Digital Object Identifier 10.1109/TAP.2009.2021882 formulations are not capable of analyzing UWB wave propagation in frequency dependent lossy environments because media parameters are specified as frequency independent constants, whilst these parameters vary with frequency in the real world. To overcome this problem, a variety of methods to include the frequency dependent materials in FDTD [1], [2] have been proposed.

In FDTD, accurate numerical modelling techniques and validation of the code are essential in order to trust the results from the numerical simulation. One of the important components in the development of FDTD schemes is the method used to introduce the main source of energy into the FDTD space, because the model of the source has a significant impact on the transient waveform produced by the simulation. Apart from the emerging class of FDTD wave sources with resistive or capacitive impedance, the punctual sources are usually implemented in FDTD grids as either a hard or a soft source [3]. When a field that excites the FDTD space needs to be specified, a hard source has advantages from the perspective of ease of implementation [4]. It simply consists of impressing an electric field component at one FDTD node.

Since the FDTD cell is shorter than one-tenth of the main wavelengths of interest, physically the soft source current acts as a Hertzian dipole antenna. Therefore, the field radiated by the soft source can be easily computed analytically by using the well known formulae of the Hertzian dipole. Conversely, for the hard source, which physically corresponds to a voltage source in the FDTD grid, no analytical solution has been reported in the literature. This is a drawback with regards to using a hard source for the validation of FDTD codes or FDTD schemes. Specifically, without an analytical solution it is difficult to test and assess accuracy of the field radiated by a hard source in Debye media in view of future calculations in such materials. This is why we have investigated and derived the analytical solution for the FDTD hard source.

Section II shows that the field radiated by the hard source is identical to the field radiated by the soft source, provided that a certain relationship holds between the source excitations. More precisely, for the two radiated fields to be identical, the hard source excitation must be the integral across time of the soft source excitation, multiplied by a coefficient that depends on the FDTD cell size. This permits analytical calculation of the hard source field, and then use of the hard source to test and assess accuracy of propagation in FDTD schemes.

Several numerical experiments to assess accuracy of the field radiated by punctual sources in FDTD grids have been reported in the literature. However, the quantification of the deviation from the analytical solution is rarely performed in these works. 
Some studies such as [2], [5] use the plot of the waveforms from both analytical solutions and results from numerical experiments without quantification. Although [4] quantified the error using the analytical solution, the quantification formula has utilised peak values of the electric field magnitudes from analytical solutions and numerical simulations. This does not provide the level of accuracy in terms of deviation of the waveform observed in the numerical simulation from the analytical solution. There is no work which quantifies accuracy of field waveforms radiated by punctual sources in the dispersive media which have to be dealt with especially in UWB systems.

Section IV reports various numerical experiments with both hard and soft sources. Conversely to most previous works, this paper uses the analytical solutions of the field radiated by the two sources presented in Section III as reference solutions with which the FDTD solutions are compared. Experiments have been performed in both vacuum and Debye media. Accuracy of the radiated field is studied by means of a criterion that utilises the whole time-domain waveform of the radiated pulse. A modulated Gaussian pulse excitation is used with the hard source, and its derivative with the soft source. The reported experiments reveal the influence of the FDTD simulation parameters, the pulse excitation parameters, and the Debye medium parameters, on the accuracy of the radiated field propagating in the FDTD grid. At the end, some criteria to be used for accurate FDTD simulations in Debye media are derived.

\section{SOURCE EXCITATION}

\section{A. Review of the Implementation of Hard and Soft Sources}

To radiate an electromagnetic wave from a single point $\left(i_{s r c} \Delta x, j_{s r c} \Delta y, k_{s r c} \Delta z\right)$ of the FDTD grid in Cartesian coordinate with $\Delta x, \Delta y, \Delta z$ the spatial step sizes in $x, y, z$ directions, respectively, the hard source method simply consists of impressing a field component, for instance, $E_{z}^{n}\left(i_{s r c}, j_{s r c}, k_{s r c}\right)$, at each time step of $n \Delta t$ as follows:

$$
E_{z}^{n}\left(i_{s r c}, j_{s r c}, k_{s r c}\right)=E_{\mathrm{S}}\left(t_{n}\right)
$$

where $\Delta t$ is the temporal step size and $E_{\mathrm{S}}\left(t_{n}\right)$ is a given function of time.

Another way to radiate a wave from a single point is to place a Hertzian dipole antenna at an $E$ node, by impressing a current $\mathcal{I}_{z}$ at this node

$$
\mathcal{I}_{z}^{n+1 / 2}\left(i_{s r c}, j_{s r c}, k_{s r c}\right)=I_{\mathrm{S}}\left(t_{n+1 / 2}\right)
$$

where $I_{\mathrm{S}}\left(t_{n+1 / 2}\right)$ is also a given function of time. Using Ampere's law and Ohm's law

$$
\frac{\partial H_{y}}{\partial x}-\frac{\partial H_{x}}{\partial y}-J_{z}=\epsilon \frac{\partial E_{z}}{\partial t}
$$

where $\epsilon$ is the permittivity, and replacing $\mathcal{I}_{z}^{n+1 / 2}\left(i_{s r c}, j_{s r c}, k_{s r c}\right)$ in the FDTD cell with the current density $J_{z}=\mathcal{I}_{z}\left(i_{s r c}, j_{s r c}, k_{s r c}\right) / \Delta x \Delta y$, the FDTD

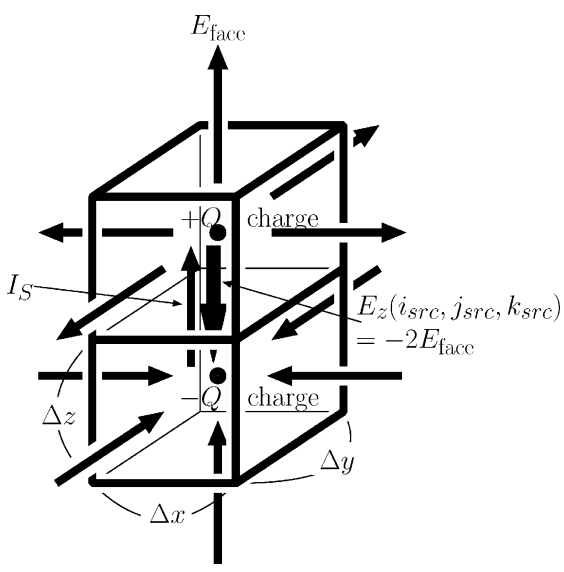

Fig. 1. Charges and fields generated in two contiguous cells by a current impressed at a FDTD node as a point source.

equation for the advance of $E_{z}$ at location $\left(i_{s r c}, j_{s r c}, k_{s r c}\right)$ reads as follows:

$$
\begin{aligned}
& E_{z}^{n+1}\left(i_{s r c}, j_{s r c}, i_{s r c}\right)=E_{z}^{n}\left(i_{s r c}, j_{s r c}, i_{s r c}\right) \\
& +\frac{\Delta t}{\epsilon}\left[\frac{\partial H_{y}}{\partial x}-\frac{\partial H_{x}}{\partial y}-\frac{\mathcal{I}_{z}^{n+1 / 2}\left(i_{s r c}, j_{s r c}, k_{s r c}\right)}{\Delta x \Delta y}\right] .
\end{aligned}
$$

This implementation, referred to as the soft source, simulates a Hertzian dipole antenna with current $\mathcal{I}_{z}$ in (2) and length $\Delta z$. As observed in experiments in [6], [7], the field radiated using (4) agrees very well with the field computed using the product $I_{\mathrm{S}}(t) \cdot \Delta z$ in the analytic dipole formulae [8].

\section{B. Comparison of the Fields Radiated by the Hard Source and the Soft Source}

Section II-B shows that the fields radiated in a FDTD grid by the hard source and the soft source are identical, provided that a relationship holds between excitations $E_{\mathrm{S}}\left(t_{n}\right)$ in (1) and $I_{\mathrm{S}}\left(t_{n+1 / 2}\right)$ in (2). To do this, let us assume that there is no incident field from other sources, i.e., the field around the source is the only radiated field. From (3) and (4) it is apparent that the soft source field at a source location is the integral in time of current and $\nabla \times \mathbf{H}$ terms. More precisely, because $\mathbf{H}$ radiated from the soft source is proportional to the impressed current, $E_{z}^{n}\left(i_{s r c}, j_{s r c}, k_{s r c}\right)$ can be expressed as

$$
E_{z}^{n}\left(i_{s r c}, j_{s r c}, k_{s r c}\right)=-\frac{1}{\epsilon_{0} \Delta x \Delta y}(1+a) \int_{-\infty}^{t_{n}} I_{\mathrm{S}}(\mathfrak{t}) d \mathfrak{t}
$$

where $a$ is an unknown coefficient and $\epsilon_{0}$ is the permittivity in vacuum. From (1) and (5), the soft source is equivalent to a hard source whose impressed field $E_{\mathrm{S}}(t)$ is the integral of the impressed current $I_{\mathrm{S}}(t)$, multiplied with coefficient in (5).

Notice that (5) is consistent with physics. If a current flows through a FDTD cell, charges of opposite signs proportional to the integral of the current are created in the adjacent cells as depicted in Fig. 1. The voltage between the charges and the $E_{z}$ field are then proportional to the integral of the current.

In order to find the unknown coefficient in (5), consider Fig. 1 and assume that a uniform spatial discretization is applied to the FDTD space, that is, $\Delta x=\Delta y=\Delta z \equiv \Delta s$. In the upper 
cell where a charge $+Q$ is enclosed, the $\mathbf{E}$ field is the same on the six faces of the cell due to symmetry. Denoting as $E_{\text {face }}$ the modulus of the field normal to the faces and applying the Gauss theorem to the surface enclosing the cell [9], [10], that is, $6(\Delta s)^{2}$,

$$
\begin{aligned}
Q & =2 \epsilon_{0} E_{\text {face }} \Delta x \Delta y+2 \epsilon_{0} E_{\text {face }} \Delta y \Delta z+2 \epsilon_{0} E_{\text {face }} \Delta z \Delta x \\
& =6 \epsilon_{0} E_{\text {face }}(\Delta s)^{2}
\end{aligned}
$$

is obtained. The lower cell with a charge $-Q$ creates a field opposite to the case for $+Q$. Finally, adding the two contributions in the separation between the two cells, the $E_{z}$ field at node $\left(i_{s r c}, j_{s r c}, k_{s r c}\right)$ is

$$
E_{z}^{n}\left(i_{s r c}, j_{s r c}, k_{s r c}\right)=-2 E_{\text {face }}=-\frac{Q\left(t_{n}\right)}{3 \epsilon_{0}(\Delta s)^{2}} .
$$

Notice that (7) can be rewritten as $V=Q / C$ where $V=E_{z} \Delta s$ is the voltage between the contiguous cells and $C=3 \epsilon_{0} \Delta s$ is the cell capacitance defined in [9], [10]. Replacing the charge $Q$ in (7) with the integral of the current in the dipole, $E_{z}$ is then

$$
E_{z}^{n}\left(i_{s r c}, j_{s r c}, k_{s r c}\right)=-\frac{1}{3 \epsilon_{0}(\Delta s)^{2}} \int_{-\infty}^{t_{n}} I_{\mathrm{S}}(\mathfrak{t}) d \mathfrak{t}
$$

This is of the expected form shown in (5), with $a=-2 / 3$. Finally, the hard source and the soft source will produce the same field at the $E_{z}\left(i_{s r c}, j_{s r c}, k_{s r c}\right)$ node, and will radiate the same field in the surrounding space, provided that

$$
E_{\mathrm{S}}(t)=-\frac{1}{3 \epsilon_{0}(\Delta s)^{2}} \int_{-\infty}^{t} I_{\mathrm{S}}(\mathfrak{t}) d \mathfrak{t}
$$

Results of FDTD experiments are provided in Fig. 2. Two FDTD calculations are performed, with the hard source and with the soft source, successively. The impressed hard source field $E_{\mathrm{S}}(t)$ is the following Gaussian pulse:

$$
E_{\mathrm{S}}(t)=10^{5} \exp \left(-\left(\frac{t-3 t_{0}}{t_{0}}\right)^{2}\right)
$$

where $t_{0}$ is $2 \mathrm{~ns}$. The impressed soft source current $I_{\mathrm{S}}(t)$ is the derivative of (10) multiplied with $3 \epsilon_{0}(\Delta s)^{2}$, in order that (9) holds. The computed $E_{z}$ field $50 \mathrm{~cm}$ (10 FDTD cells) from the dipole is shown in Fig. 2. In addition to hard source and soft source results, the analytical solution is also plotted in Fig. 2. As observed, the three results are in good agreement. Both hard source and soft source yield results about superimposed with the analytical formulae of the Hertzian dipole antenna. So, in the absence of an incident field around the source, hard source and soft source are equivalent provided that (9) holds.

Obviously, in the case where an incident field radiated by other sources is present, the two sources are not equivalent, because $E_{z}$ is impressed at the hard source location so that the incident field is enforced as zero at this location. For the incident field the hard source acts as a PEC, that is a zero impedance boundary condition. Finally, if an incident field is present, the hard source is equivalent to a soft source plus a PEC condition. This is in accordance with the interpretation of the hard source as a voltage source with zero impedance that can be found in the literature[3].

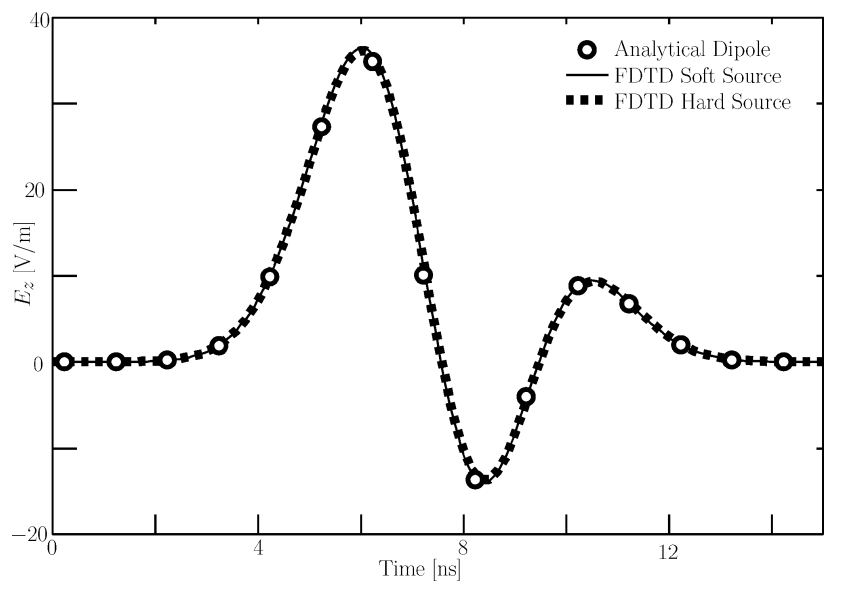

Fig. 2. Electric field $50 \mathrm{~cm}$ from a short dipole, computed with the 3D FDTD method and the soft source, the 3D FDTD method and the hard source, and analytical formulae. The FDTD cell is a cube of size $\Delta s=5 \mathrm{~cm}$, the time step $\Delta t$ is 83.3 ps with the FDTD space surrounded by a PML ABC [12].

In deriving (9), it is has been assumed that the FDTD cell is cubic. With a parallelepipedic cell the fields on the six faces are not equal, and then (9) is questionable. Nevertheless, (5) is still valid, so that the hard source remains equivalent to a soft source. Therefore, for the radiated fields to be equal, (9) is still valid, but with a coefficient that would differ from $1 / 3 \epsilon_{0}(\Delta s)^{2}$.

\section{Hard Source in Non-Vacuum}

The results in Section II-B have been derived in a vacuum. They can be extended to sources placed in other media. In a dissipative medium of conductivity $\sigma$ by taking account of the conduction current density $\sigma E_{z},(8)$ is replaced with

$$
\begin{aligned}
E_{z}^{n}\left(i_{s r c}, j_{s r c}, k_{s r c}\right)+\frac{\sigma}{3 \epsilon_{0}} \int_{-\infty}^{t_{n}} E_{z}(\mathfrak{t}) d \mathfrak{t} & = \\
& -\frac{1}{3 \epsilon_{0}(\Delta s)^{2}} \int_{-\infty}^{t_{n}} I_{\mathrm{S}}(\mathfrak{t}) d \mathfrak{t} .
\end{aligned}
$$

In the case where $\sigma \gg 3 \epsilon_{0} \omega$, where $\omega$ is the angular frequency, using the frequency domain counterpart of (11), it can be easily shown that (9) is replaced by

$$
E_{\mathrm{S}}(t)=-\frac{1}{\sigma(\Delta s)^{2}} I_{\mathrm{S}}(t) .
$$

This is nothing but Ohm's law, since if both sides are multiplied by $\Delta s$ the left hand-side is a voltage and the inverse of $\sigma \Delta s$ is a resistor. In the case of more general media, such as the Debye media addressed in Section III, this reasoning is still valid with $\epsilon_{0}$ removed from (6), (7), and (8) and $\mathbf{E}$ field replaced with flux density $\mathbf{D}$. Then, $D_{\mathrm{S}}(t)$ at the source location if the hard source radiate the same field as the soft source is

$$
D_{\mathrm{S}}(t)=-\frac{1}{3(\Delta s)^{2}} \int_{-\infty}^{t} I_{\mathrm{S}}(\mathfrak{t}) d \mathfrak{t} .
$$

The electric field $E_{\mathrm{S}}(t)$ to be impressed can be obtained from

$$
\mathbf{D}=\epsilon \mathbf{E}
$$

by means of the algorithm used at the regular nodes of the grid, for instance, that given in [11]. 


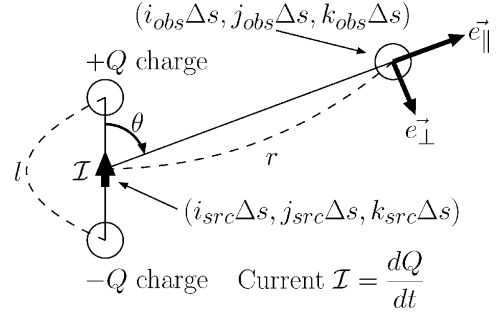

Fig. 3. The definition of the unit vectors $\overrightarrow{\boldsymbol{e}_{\|}}$and $\overrightarrow{\boldsymbol{e}_{\perp}}$ relative to the location of the dipole.

\section{The Analytical Solution of Field Distribution From a Dipole IN DEBye Media}

Among a variety of frequency dependent models, this paper adopts the classical and most popular type, the Debye model[13]. In the first order Debye model, the relative permittivity $\epsilon_{\text {debye }}$ becomes a complex number such as

$$
\epsilon_{\text {debye }}(f)=\epsilon_{\infty}+\frac{\epsilon_{\mathrm{S}}-\epsilon_{\infty}}{1+\jmath 2 \pi f \tau_{\mathrm{D}}}
$$

where $\jmath=\sqrt{-1}, \epsilon_{\infty}, \epsilon_{\mathrm{S}}$, and $\tau_{\mathrm{D}}$ are the optical permittivity, the static permittivity and the relaxation time, respectively.

When the soft source sinusoidally varies in time in a vacuum, $\mathbf{E}$ around the dipole is analytically known[14]. [2] presents the analytical solution of a pulse excitation without clear explanation of the inclusion of the effect of the medium in the analytical solution. [5] obtains the analytical solution by convolution of the quasi-static impulse response of the FDTD space and the frequency spectrum of source excitation. Instead, this paper explicitly includes the first order Debye media into the analytical solution. In a lossy medium, each frequency component of an UWB signal $\mathcal{S}(f)$ has its own propagation speed. Taking this into account, the analytical $\mathbf{E}$ in a vacuum at the observation point $\left(i_{o b s} \Delta x, j_{o b s} \Delta y, k_{o b s} \Delta z\right)$ is modified to obtain the analytical $\mathbf{D}$ in a Debye medium, noted as $\mathcal{D}_{\boldsymbol{s}} . \mathcal{D}_{\boldsymbol{s}}$ is written as

$$
\begin{aligned}
\mathcal{D}_{\boldsymbol{s}}(\mathcal{S}(f))= & \epsilon_{0} \epsilon_{r} \mathcal{S}(f) l \frac{e^{-2 \jmath \pi f r \sqrt{\mu \epsilon_{0} \epsilon_{r}}}}{4 \jmath \pi^{2} f \epsilon_{0} \epsilon_{r}} \\
& \times\left(\frac{1}{r^{3}}+\frac{2 \jmath \pi f \sqrt{\mu \epsilon_{0} \epsilon_{r}}}{r^{2}}\right) \cos (\theta) \overrightarrow{\boldsymbol{e}_{\|}} \\
& +\epsilon_{0} \epsilon_{r} \mathcal{S}(f) l \frac{e^{-2 \jmath \pi f r \sqrt{\mu \epsilon_{0} \epsilon_{r}}}}{8 \jmath \pi^{2} f \epsilon_{0} \epsilon_{r}} \\
& \times\left(\frac{1}{r^{3}}+\frac{2 \jmath \pi f \sqrt{\mu \epsilon_{0} \epsilon_{r}}}{r^{2}}-\frac{4 \pi^{2} f^{2} \mu \epsilon_{0} \epsilon_{r}}{r}\right) \sin (\theta) \overrightarrow{\boldsymbol{e}_{\perp}}
\end{aligned}
$$

where

$$
\epsilon_{r}=\epsilon_{\text {debye }}(f)+\frac{\sigma}{\jmath 2 \pi f \epsilon_{0}}
$$

$r$ is the distance between the source and observation points, $\overrightarrow{\boldsymbol{e}_{\|}}$is the unit vector in the direction from the source to the observation point, and the unit vector $\overrightarrow{\boldsymbol{e}}$ is perpendicular to $\overrightarrow{\boldsymbol{e}_{\|}}$as shown in Fig. 3.

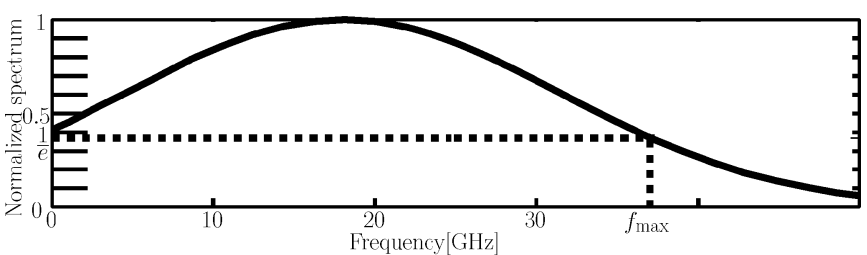

Fig. 4. The source excitation spectrum and the definition of $f_{\max }$.

This analytical solution $\mathcal{D}_{\boldsymbol{s}}(\mathcal{S}(f))$ is used for the accuracy assessment of both soft and hard source excitations.

\section{ACCURACY ASSESSMENT}

\section{Definition of the Deviation}

The deviation $\mathcal{E}$ of the observed waveform in the FDTD space from the analytical waveform can be quantified as follows:

$$
\mathcal{E}=\sqrt{\frac{\sum_{f}\left\{\mathcal{F}\left(g_{O}(t)\right)-\mathcal{D}_{\boldsymbol{s}}(\mathcal{S}(f))\right\}^{2}}{\sum_{f}\left|\mathcal{D}_{\boldsymbol{s}}(\mathcal{S}(f))\right|^{2}}}
$$

where $g_{O}(t)$ is the signal observed in the FDTD space and $\mathcal{F}\left(g_{O}(t)\right)$ is the frequency spectrum of $g_{O}(t) . \mathcal{E}$ quantifies the waveform degradation from the analytical solution, not the stability measurement. All of the calculations performed in this paper are stable. This frequency domain assessment takes into account the waveform deviation, the difference in time of arrival, and the amplitude difference from the analytical solution. $\mathcal{E}$ changes over the propagation distance.

The dimensions of the FDTD space are usually governed by the available memory in the computer, independent of $\Delta s$. In this case, $\mathcal{E}$ is discussed as a function of the number of grids between the source excitation and the observation. Therefore, this paper assesses $\mathcal{E}$ over the propagation distance in a FDTD cell rather than the physical propagation distance.

\section{A. Settings for Numerical Experiments}

A $360 \times 360 \times 360$ dimensional FDTD space is excited at its center with the modulated Gaussian pulse of

$$
D_{\mathrm{S}}(t)=\exp \left(-\left(\frac{\pi t}{T_{\mathrm{G}}}\right)^{2}\right) \cos \left(2 \pi \frac{t}{T_{\mathrm{c}}}\right)
$$

where $T_{\mathrm{G}}$ is a parameter for a Gaussian pulse width and $1 / T_{\mathrm{C}}$ is a carrier frequency. The spectrum of the modulated Gaussian pulse is plotted in Fig. 4.

This paper defines the highest frequency of interest whose spectrum is $1 / e$ relative to the spectrum maximum as $f_{\max }$. $f_{\max }$ is expressed using $T_{\mathrm{G}}$ and $T_{\mathrm{c}}$ as follows:

$$
f_{\max }=\frac{1}{T_{\mathrm{G}}}+\frac{1}{T_{\mathrm{c}}} \text {. }
$$


TABLE I

PARAmeters to Set the Source EXCITATION SHOWN IN FIG. 4 WHICH ARE USED ThroughouT IN SECTIONS IV-B AND IV-C

\begin{tabular}{|c|c|c|c|c|}
\hline$T_{\mathrm{G}}$ & $T_{\mathrm{c}}$ & $f_{\max }$ & $\lambda_{\min }$ & centre frequency \\
\hline$\frac{\pi}{60} \mathrm{~ns}$ & $\frac{1}{18} \mathrm{~ns}$ & $37.1 \mathrm{GHz}$ & $8.09 \mathrm{~mm}$ & $18 \mathrm{GHz}$ \\
\hline
\end{tabular}

The wavelength $\lambda_{\min }$ corresponding to $f_{\max }$ is $v / f_{\max }$, where $v$ is the propagation speed in vacuum. The frequency at which the frequency spectrum has its peak magnitude is defined as a center frequency. Sections IV-B and IV-C set $T_{\mathrm{G}}, T_{\mathrm{c}}, f_{\max }, \lambda_{\min }$ and center frequency to constant values presented in Table I whilst $\Delta s$ and $\epsilon_{r}$ vary. The factor

$$
\frac{\lambda_{\min }}{\Delta s \sqrt{\epsilon_{r}}}
$$

is used to characterise the propagation behavior of the pulse in a Debye medium. When $\epsilon_{r}$ is frequency dependent, the real part of $\epsilon_{r}$ at the center frequency of the source excitation is used for the calculation of $\sqrt{\epsilon_{r}}$ in (21). This paper studies the propagation of the radiated field along the $x$-axis of the FDTD domain, from its center where the source is placed towards the outer boundary. The propagation path corresponds to the propagation along $r$ with $\theta=90^{\circ}$ in Fig. 3. The FDTD space is large enough to capture the line-of-sight signal and stop the observation well before a first reflection from the artificial boundary arrives at the observation points. From this, the FDTD experiments simulate the radiation of the source in an infinite homogeneous medium.

The FDTD space is filled with one of the following media:

- Medium 1 Vacuum.

- Medium 2 Debye medium whose parameters are $\left(\sigma, \epsilon_{\infty}, \epsilon_{\mathrm{S}}, \tau_{\mathrm{D}}\right)=(0.2 \mathrm{~S} / \mathrm{m}, 2.8,4.8,7 \mathrm{ps}) \cdot \epsilon_{r}$ is 4 at the center frequency and Medium 2 has a loss tangent while Medium 1 does not have a loss tangent.

$\mathcal{E}$ is expected to increase with the decrease of $\lambda_{\min } / \Delta s \sqrt{\epsilon_{r}}$. Section IV-C examines this expectation computationally.

\section{B. Numerical Experiment Results}

1) Relationship of $\mathcal{E}$ and $\lambda_{\min } / \Delta s \sqrt{\epsilon_{r}}$ : Section IV-C.1 uses Medium 1 as the simplest case to examine the method of quantifying the numerical noise and the effect of $\lambda_{\min } / \Delta s \sqrt{\epsilon_{r}}$ on $\mathcal{E}$. The numerical experiments are performed using the soft source excitation, varying $\lambda_{\min } / \Delta s \sqrt{\epsilon_{r}}$ from 3 to 20 by changing $\Delta s$ with a constant $\lambda_{\min } \simeq 8.09 \mathrm{~mm}$ which corresponds to $f_{\max }$ of $37.1 \mathrm{GHz}$. Fig. 5 gives the time domain signals observed for $\lambda_{\min } / \Delta s \sqrt{\epsilon_{r}}$ equal to $6.1,8.5$ or 12.1. For all cases, two observation locations are set; one at $1 / 75$ meters (about $1.65 \lambda_{\min }$ ) and the other at 1/10 meters (about $12.4 \lambda_{\min }$ ) away from the source. In order to avoid the inclusion of spurious reflections, which originates at the boundary, the leading edge of the signals reflected off the boundary reaches these observation points after the trailing edge of the line-of-sight signal from the point source fades away. The signal observed is time-gated only for the line-of-sight signals. The analytical solution is also plotted as a reference. The analytical solution at each observation is normalized and the observation is plotted relative to the normalized analytical solution. The numerical noise accumulation over the propagation distance is clearly observed for all three

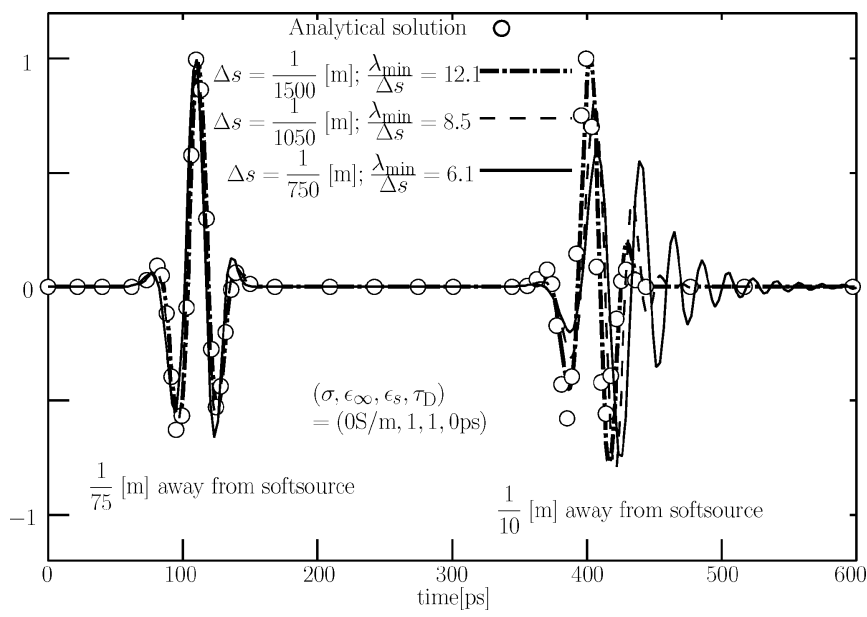

Fig. 5. Signals from the soft source in the FDTD space in Medium 1 (vacuum) when $\lambda_{\min } / \Delta s \sqrt{\epsilon_{r}}$ is either $6.1,8.5$ or 12.1 . In the case of $\lambda_{\min } / \Delta s \sqrt{\epsilon_{r}}=6.1$, the observation points are placed at $10 \Delta s$ and $75 \Delta s$ away from the source. For $\lambda_{\min } / \Delta s \sqrt{\epsilon_{r}}=8.5$, the signals at $14 \Delta s$ and $105 \Delta s$ away from the source are observed. For $\lambda_{\min } / \Delta s \sqrt{\epsilon_{r}}=12.1$, the signals at $20 \Delta s$ and $150 \Delta s$ away from the source are observed. Strong numerical noise is observed for lower $\lambda_{\min } / \Delta s \sqrt{\epsilon_{r}}$ at longer propagation distance.

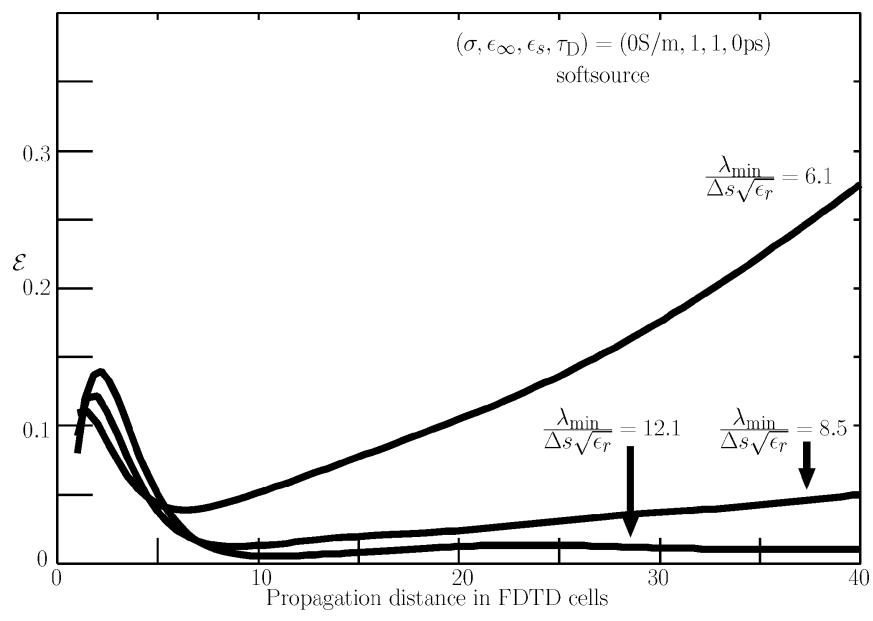

Fig. 6. $\mathcal{E}$ which is the function of $\lambda_{\min } / \Delta s \sqrt{\epsilon_{r}}$ and the propagation distance $\left|i_{s r c}-i_{o b s}\right|$ from the soft source in Medium 1 . The characteristics of the numerical noise observed in Fig. 5 is quantified. $\mathcal{E}$ with $\lambda_{\min } / \Delta s \sqrt{\epsilon_{r}}>12.1$ is comparable to $\mathcal{E}$ with $\lambda_{\min } / \Delta s \sqrt{\epsilon_{r}}=12.1$.

cases of $\lambda_{\min } / \Delta s \sqrt{\epsilon_{r}}$. The deviation of the signal from the analytical solution at about $12.4 \lambda_{\min }$ away from the source with $\lambda_{\min } / \Delta s \sqrt{\epsilon_{r}}=6.1$ is larger than the case with $\lambda_{\min } / \Delta s \sqrt{\epsilon_{r}}=$ 8.5. This phenomenon is clearly presented in Fig. 6. $\mathcal{E}$, which quantifies the difference between the analytical solution and the observation, is demonstrated in the cases of $\lambda_{\min } / \Delta s \sqrt{\epsilon_{r}}=$ 6.1, 8.5, and 12.1 in Fig. 6. A high $\mathcal{E}$ is observed in the vicinity of the source excitation for all the cases. This result matches to [4] in that the high error region is within a fixed number of cells of the source point. The decrease of $\lambda_{\min } / \Delta s \sqrt{\epsilon_{r}}$ and the increase of the propagation distance give higher $\mathcal{E}$. $\mathcal{E}$ when $\lambda_{\min } / \Delta s \sqrt{\epsilon_{r}}>12.1$ is comparable to and lower than $\mathcal{E}$ in the case of $\lambda_{\min } / \Delta s \sqrt{\epsilon_{r}}=12.1$ although they are not shown in Fig. 6.

2) Relationship of $\mathcal{E}$ and Media: The characteristics of the wave propagation differ depending on the media, even when 


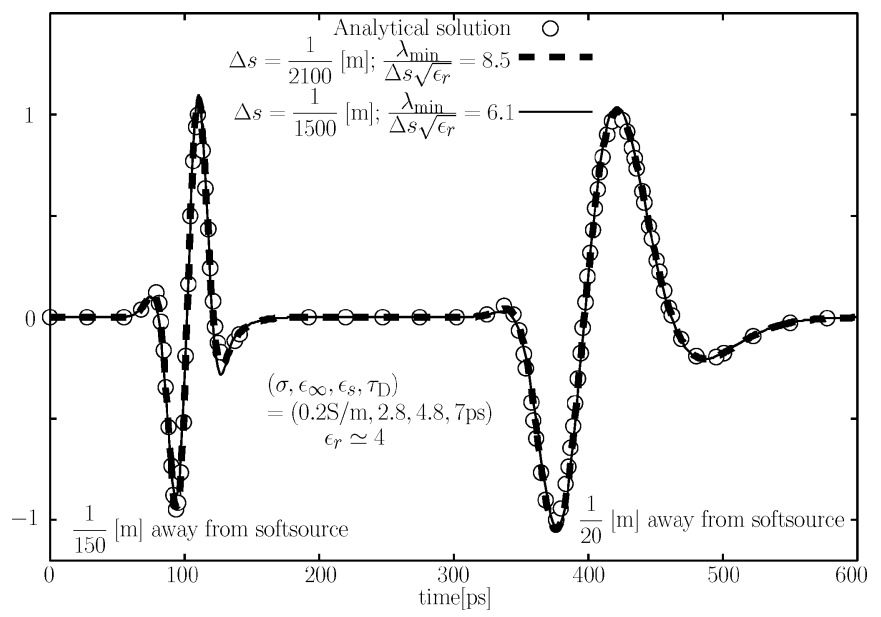

Fig. 7. Signals from the soft source in the FDTD space with Medium 2 when $\lambda_{\min } / \Delta s \sqrt{\epsilon_{r}}$ is either 6.1 or 8.5 . The result for $\lambda_{\min } / \Delta s \sqrt{\epsilon_{r}}=12.1$ is not plotted as it is almost identical to the analytical solution.

$\lambda_{\min } / \Delta s \sqrt{\epsilon_{r}}$ is identical between the different media when $\lambda_{\min } / \Delta s \sqrt{\epsilon_{r}}<10$. Section IV-C.2 deals with soft source and Medium 2. Fig. 7 shows time domain signals observed in the case of $\lambda_{\min } / \Delta s \sqrt{\epsilon_{r}}=8.5$ and 6.1. The same normalization procedure is taken as in Fig. 5. The distance between the source excitation and observations is the same as Fig. 5 in FDTD cells (not physical distance). The observation locations are $10 \Delta s$ and $75 \Delta s$ in case of $\lambda_{\min } / \Delta s \sqrt{\epsilon_{r}}=6.1$ and the locations $14 \Delta s$ and $105 \Delta s$ away from the source are observed in the case of $\lambda_{\min } / \Delta s \sqrt{\epsilon_{r}}=8.5$. No significant deviation from the analytical solution is observed for both cases, $\lambda_{\min } / \Delta s \sqrt{\epsilon_{r}}=6.1$ and 8.5, at both observation locations of $1 / 150 \mathrm{~m}$ and $1 / 20 \mathrm{~m}$ away from the source. Even when $\lambda_{\min } / \Delta s \sqrt{\epsilon_{r}}$ is as low as 6.1 , no high numerical noise such as that in Fig. 5, is observed and the observation has a reasonable match to the analytical solution. The signals observed at the locations physically the same to those in Fig. 5 for both cases are almost superimposed on the analytical solutions, although they are not plotted in Fig. 7.

As is seen in Fig. 5, the numerical noise tends to involve spurious high frequency components. However, if a medium has a loss tangent, the high frequency components caused by numerical noise seem to be absorbed. This phenomenon is clearly quantified in Fig. 8. $\mathcal{E}$ is plotted for $\lambda_{\min } / \Delta s \sqrt{\epsilon_{r}}=6.1,8.5$ and 12.1. Although $\mathcal{E}$ increases with the decrease of $\lambda_{\min } / \Delta s \sqrt{\epsilon_{r}}$, $\mathcal{E}$ for all three cases are fairly comparable and a significant increase of $\mathcal{E}$ over the propagation distance is not observed. This could be caused by the media's absorption of the high frequency components produced by the numerical dispersion.

3) $\mathcal{E}$ Difference Between the Soft and Hard Source Excitations: The comparison between $\mathcal{E}$ for the soft source and $\mathcal{E}$ for the hard source is performed varying $\lambda_{\min } / \Delta s \sqrt{\epsilon_{r}}$ in Medium 1 and Medium 2. $\mathcal{E}$ from the hard source in Medium 1 is plotted in Fig. 9. When $\lambda_{\min } / \Delta s \sqrt{\epsilon_{r}}$ is $12.1, \mathcal{E}$ in Fig. 6 is comparable to $\mathcal{E}$ in Fig. 9 and both are less than $3 \%$ in error at $40 \Delta s$ away from the source. However, $\mathcal{E}$ of the hard source for $\lambda_{\min } / \Delta s \sqrt{\epsilon_{r}}<10$ is more than double of $\mathcal{E}$ of the soft source. Therefore, it can be safely concluded that $\lambda_{\min } / \Delta s \sqrt{\epsilon_{r}}$ should be at least of the order of 10 to have negligible numerical noise.

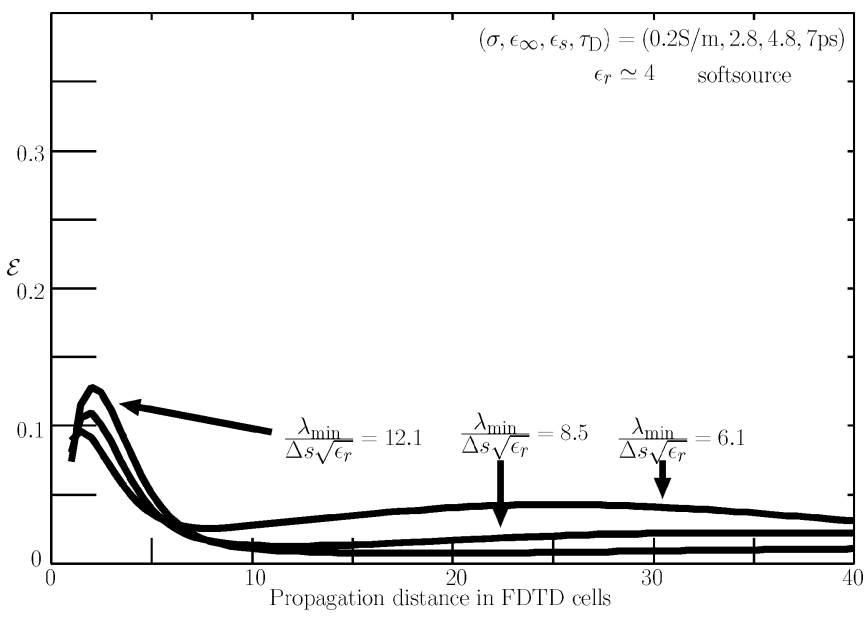

Fig. 8. $\mathcal{E}$ from the soft source in Medium 2. The characteristics of the numerical noise observed in Fig. 7 are presented. $\mathcal{E}$ with $\lambda_{\min } / \Delta s \sqrt{\epsilon_{r}}>12.1$ is comparable to $\mathcal{E}$ with $\lambda_{\min } / \Delta s \sqrt{\epsilon_{r}}=12.1$. The decrease of $\mathcal{E}$ over the propagation distance and low $\mathcal{E}$ with $\lambda_{\min } / \Delta s \sqrt{\epsilon_{r}}<10$ are the noticeable difference from Fig. 6.

In Medium 2, the signal from the hard source has a similar tendency to that from the soft source. Fig. 10 shows the signals from both a hard and soft source in Medium 2. As a reference, the analytical solution is also plotted. At $1 / 150 \mathrm{~m}$ away from the source, the signal from the hard source has a significantly larger deviation from the analytical solution than that from the soft source. On the other hand, the signals from both sources are comparable at $1 / 20 \mathrm{~m}$ away from the source. This rapid reduction of the deviation from the analytical solution in the case of the hard source is quantified in Fig. 11. The comparisons between Figs. 6 and 9 and the one between Figs. 8 and 11 suggest that the soft source implementation outperforms the hard source and the hard source results degrade more rapidly as the FDTD grid becomes coarser, i.e., as $\lambda_{\min } / \Delta s \sqrt{\epsilon_{r}}$ decreases. This is not surprising because the reasoning used to derive (6) and (7) assumes that $\mathbf{E}$ field and current are static quantities within the considered FDTD cell. From this, (9) is only valid for wavelengths large in comparison with the FDTD cell. If this condition does not hold, the hard source is not equivalent, rigorously, to the soft source, resulting in the larger deviations observed in Section IV-C.3 and Fig. 11 for $\lambda_{\min } / \Delta s \sqrt{\epsilon_{r}}<10$.

4) $\mathcal{E}$ Difference Depending on the Debye Media Parameters: Based on the recommendation in Section IV-C.3, Section IV-C.4 deals with the soft source exclusively. As seen in Figs. 6 and 8, the appearance of the numerical noise in a Debye medium is rather different from that in a vacuum. The absorption of the high frequency components of the spurious frequencies by the loss tangent of the medium is raised as a major reason for the difference. To verify this, Section IV-C.4 details the effect of Debye media parameters on $\mathcal{E}$ using two types of media whose difference is largely conductivity.

- Medium 3 Debye medium whose parameters are $\left(\sigma, \epsilon_{\infty}, \epsilon_{\mathrm{S}}, \tau_{\mathrm{D}}\right)=(0.01 \mathrm{~S} / \mathrm{m}, 2.8,4.8,7 \mathrm{ps})$;

- Medium 4 Debye medium whose parameters are $\left(\sigma, \epsilon_{\infty}, \epsilon_{\mathrm{S}}, \tau_{\mathrm{D}}\right)=(0.2 \mathrm{~S} / \mathrm{m}, 2.8,4.8,21 \mathrm{ps})$.

Section IV-C.4 limits the frequency range of interest from $1 \mathrm{GHz}$ to $100 \mathrm{GHz}$. The frequency range whose excitation spectrum 


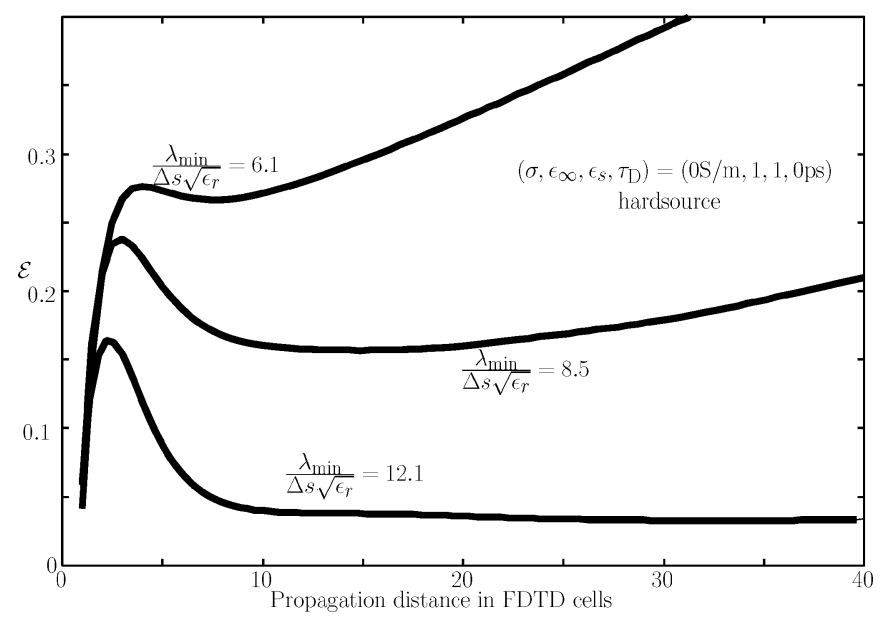

Fig. 9. $\mathcal{E}$ from the hard source in Medium 1. $\mathcal{E}$ with $\lambda_{\min } / \Delta s \sqrt{\epsilon_{r}}>12.1$ is comparable to $\mathcal{E}$ with $\lambda_{\min } / \Delta s \sqrt{\epsilon_{r}}=12.1$. The plot for $\mathcal{E}$ with $\lambda_{\min } / \Delta s \sqrt{\epsilon_{r}}=6.1$ is $1 / 3$ of the actual $\mathcal{E}$ so as to allow the vertical range to be the same as in Fig. 6 .

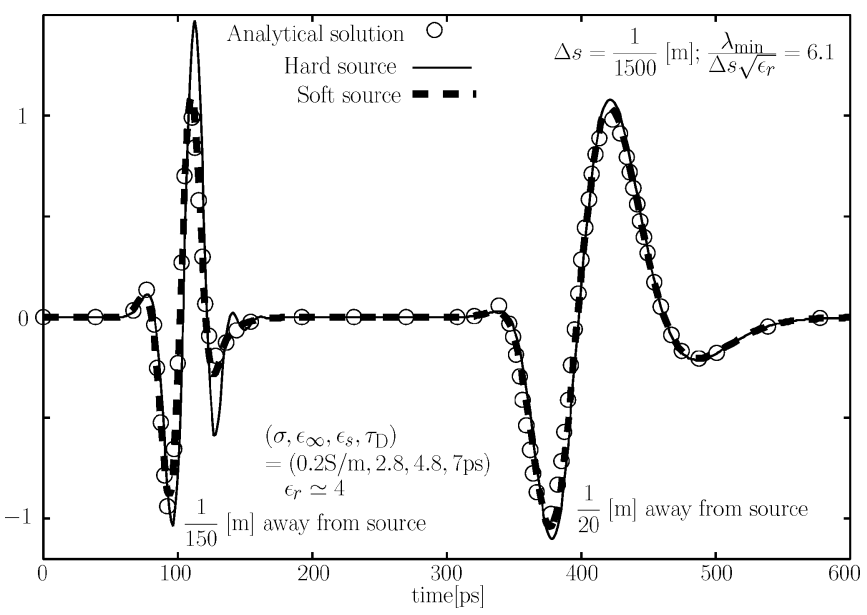

Fig. 10. Signals from the soft source and hard source in the FDTD space with Medium 2 when $\lambda_{\min } / \Delta s \sqrt{\epsilon_{r}}$ is 6.1 .

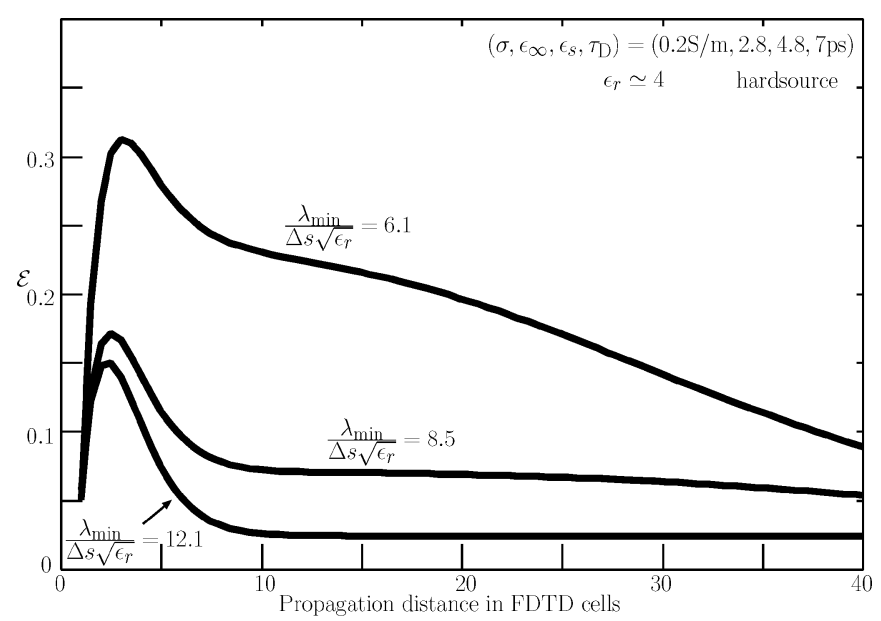

Fig. 11. $\mathcal{E}$ from the hard source in Medium 2. The characteristics of the numerical noise observed in Fig. 10 are presented.

amplitude is more than $1 / e$ relative to the highest spectrum is 1-37 GHz. The total relative permittivity and the total conductivity $[\mathrm{S} / \mathrm{m}]$ of Medium 3 and Medium 4 are plotted in Fig. 12.

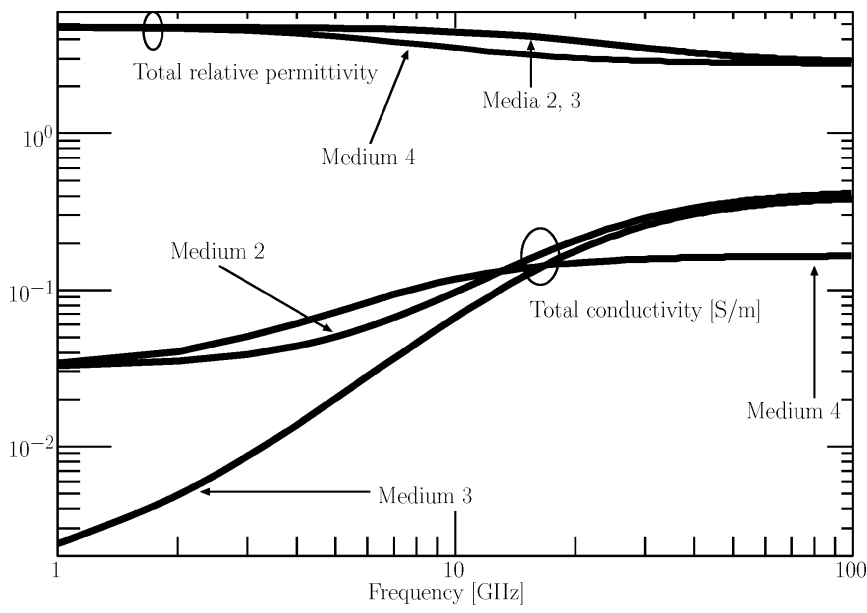

Fig. 12. The variation of the total relative permittivity and conductivity $[\mathrm{S} / \mathrm{m}]$ from $1 \mathrm{GHz}$ to $100 \mathrm{GHz}$ for Medium 3 and Medium 4.

The permittivity of Medium 3 and 4 is highly comparable to that of Medium 2 over all frequencies. The difference in Medium 2, 3 , and 4 is the frequency variation of the conductivity. At the low end of the frequency range of interest, the conductivity of Medium 3 is about $25 \mathrm{~dB}$ below the conductivity of Medium 2, but the conductivities of these two media are almost identical above $18 \mathrm{GHz}$. On the other hand, Medium 4 has a very comparable level of conductivity to Medium 2 below $18 \mathrm{GHz}$, but at the high end of the frequency range of interest, the conductivity of Medium 4 is about $7 \mathrm{~dB}$ less than that of Medium 2.

Medium 4 which has low conductivity in the high frequency region is expected to absorb less spurious frequency components than Medium 2, leading to the high $\mathcal{E}$. Since the spurious frequency components do not occur in the low frequency region, the low loss tangent, i.e., low conductivity in the low frequency region should not affect $\mathcal{E}$ significantly.

The effect of the conductivity difference on $\mathcal{E}$ is shown in Fig. 13 which presents $\mathcal{E}$ for Media 2, 3 and 4 when $\lambda_{\min } / \Delta s \sqrt{\epsilon_{r}}$ is 6.1. As expected from the above discussion, $\mathcal{E}$ for Medium 3 is highly similar to $\mathcal{E}$ for Medium 2, but $\mathcal{E}$ for Medium 4 shows the accumulation of the numerical noise over the propagation distance and approaches $\mathcal{E}$ for the Medium which has the same permittivity as Medium 4 but no loss tangent.

Therefore, it is safely concluded that high conductivity in the high frequency range suppresses $\mathcal{E}$ by absorbing the spurious high frequency noise caused by the numerical dispersion of the FDTD mesh.

\section{CONCLUSION}

The equivalence of the fields radiated by the hard source and the soft source has been proved theoretically, providing then an analytical solution to the hard source field radiated into a FDTD space. Nevertheless, despite this theoretical equivalence, use of the soft source seems preferable when radiating energy from a punctual source toward a FDTD grid, for two reasons. Firstly, the parasitic PEC present with the hard source can make the computed results wrong if radiated or reflected fields strike the source point. Secondly, numerical experiments have shown that the accuracy of the computed radiated field is higher with the 


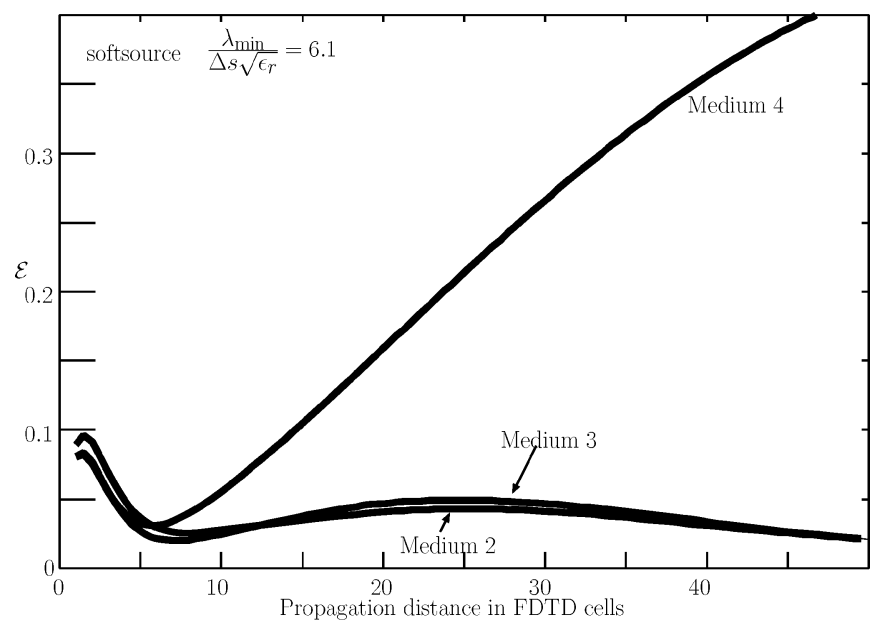

Fig. 13. $\mathcal{E}$ from the soft source in Media 2, 3 and 4. $\lambda_{\min } / \Delta s \sqrt{\epsilon_{r}}$ is 6.1 .

soft source, especially at high frequencies when the ratio of the minimum wavelength to the spatial step is approximately 10 or less.

The accuracy of the field radiated from a source in Debye media has been tested with a typical UWB waveform, i.e., a modulated Gaussian pulse. Effects dependent on the parameters of the medium and of the ratio of the minimum wavelength to the FDTD spatial sampling step, that is $\lambda_{\min } / \Delta s \sqrt{\epsilon_{r}}$, have been addressed. The field radiated from the soft source shows good matching of the FDTD solution with the analytical solution, even when the ratio $\lambda_{\min } / \Delta s \sqrt{\epsilon_{r}}$ is smaller than 10 . When frequency dependent materials, which have some loss tangent, are modeled in FDTD space, a lower $\lambda_{\min } / \Delta s \sqrt{\epsilon_{r}}$ is sufficient to achieve the same accuracy as the case in a vacuum. In the case of inhomogeneous media, the required $\lambda_{\min } / \Delta s \sqrt{\epsilon_{r}}$ is governed by the medium with the lowest conductivity at higher frequencies. Although this paper handles Debye media exclusively, the results are applicable to the other frequency dependent media such as Cole-Cole or Lorentz media. This is because the source excitation method for these alternative media is the same as the Debye case and the effect of permittivity and conductivity on the numerical accuracy should be identical, independent of the way the frequency dependent materials are implemented in FDTD.

\section{ACKNOWLEDGMENT}

The authors wish to thank Dr. M. Bonilla at EADS Nucletudes, France and Prof. C. Christopoulos at University of Nottingham, U.K. for their constructive technical discussions for the paper production, Dr. P. F. Wilson at the National Institute of Standards and Technology for his kind help to improve the manuscript, and Mr. B. Ákos at the European Grid resources (www.eu-egee.org) for the practical advice on the usage of computational resources for the numerical simulations.

\section{REFERENCES}

[1] R. J. Luebbers, F. Hunsberger, and K. S. Kunz, "A frequency-dependent finite difference time domain formulation for transient propagation in plasma," IEEE Trans. Antennas Propag., vol. 39, pp. 29-34, 1991.
[2] W. H. Weedon and C. M. Rappaport, "A general method for FDTD modeling of wave propagation in arbitrary frequency-dispersive media," IEEE Trans. Antennas Propag., vol. 45, pp. 401-410, 1997.

[3] C. E. Brench and O. M. Ramahi, "Source selection criteria for FDTD models," in Proc. IEEE Int. Symp. Electromagn. Compat., 1998, pp. 24-28.

[4] D. Buechler, D. Roper, C. Durney, and D. Christensen, "Modeling sources in the FDTD formulation and their use in quantifying sources and boundary condition errors," IEEE Trans. Microw. Theory Tech., vol. 43, pp. 810-814, 1995.

[5] D. Johnson, C. Furse, and A. C. Tripp, "FDTD modeling and validation of EM survey tool," Microw. Opt. Technol. Lett., vol. 34, pp. 427-429, 2002.

[6] J.-P. Bérenger, "Three-dimensional perfectly matched layer for the absorption of electromagnetic waves," J. Comp. Phys., vol. 127, pp. 363-379, 1996.

[7] J.-P. Bérenger, "FDTD computation of VLF-LF propagation in the Earth-Ionosphere Waveguide," Ann. Telecom, vol. 57, no. 11-12, pp. 1059-1090, 2002.

[8] J. A. Kong, Theory of Electromagnetic Waves. New York: Wiley, 1975.

[9] A. Taflove and S. Hagness, Computational Electromagnetics. The Finite-Difference Time-Domain Method. Boston, MA: Artech House, 2000.

[10] C. L. Wagner and J. B. Schneider, "Divergent fields, charge, and capacitance in FDTD simulations," IEEE Trans. Microw. Theory Tech., vol. 46, pp. 2131-2136, 1998.

[11] F. Costen and A. Thiry, "Alternative formulation of three dimensional frequency dependent ADI-FDTD method," IEICE Electron. Express, vol. 1 , pp. 528-533, 2004

[12] J.-P. Bérenger, "A perfectly matched layer for the absorption of electromagnetic waves," J. Comp. Phys., vol. 114, pp. 185-200, 1994.

[13] P. Debye, Polar Molecules. New York: Dover, 1929.

[14] B. S. Guru and H. R. Hiziroglu, Electromagnetic field Theory Fundamentals. Cambridge, U.K.: Cambridge Univ. Press, 2004.

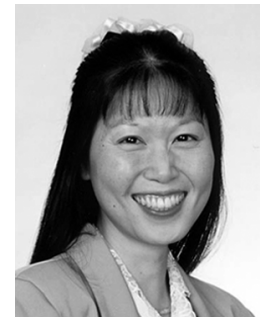

Fumie Costen (M'07) received the B.Sc. and M.Sc. degrees in electrical engineering and the Ph.D. degree in informatics, all from Kyoto University, Kyoto, Japan

From 1993 to 1997, she was with Advanced Telecommunication Research International, Kyoto, where her domain of interest was direction-of-arrival estimation based on the MUSIC algorithm for three-dimensional laser microvision. From 1998 to 2000, she was with Manchester Computing in the University of Manchester, Manchester, U.K., where she engaged in the research on metacomputing. Since 2000, she has been a Lecturer in the University of Manchester. Her main field of interest is the computational electromagnetics in such topics as finite difference time domain method for low frequency and high spatial resolution and FDTD subgridding. She was awarded three patents in 1999.

Dr. Costen received an ATR Excellence in Research Award in 1996, an academic invitation from the Kiruna Division, Swedish Institute of Space Physics, Sweden, in 1996, and a Best Paper Award from 8th International Conference on High Performance Computing and Networking Europe in 2000.

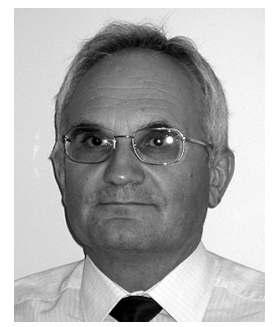

Jean-Pierre Bérenger (F'09) received a Master in Physics from the University Joseph Fourier, Grenoble, France, in 1973 and a Master in Optical Engineering from the Institut d'Optique Graduate School (formerly Ecole Supérieure d'Optique), Paris, France, in 1975.

From 1975 to 1984, he was with the Département Etudes Théoriques of the Centre d'Analyse de Défense, where his domains of interest were the propagation of waves and the coupling problems related to the nuclear electromagnetic pulse. During this period he helped popularize the finite-difference time-domain method in France. In 1984, he moved to the Département Nucléaire where he was involved in the development of simulation software. From 1989 to 1998, he held a position as expert on the electromagnetic effects of nuclear disturbances. $\mathrm{He}$ is now a Contract Manager while staying active in the field of numerical 
electromagnetics, in such topics as low frequency propagation, absorbing boundary conditions, and FDTD subgridding.

Prof. Bérenger is a member of the Electromagnetics Academy. He is currently an Associate Editor of the IEEE TRANSACTIONS ON ANTENNAS AND PROPAGATION.

Anthony K. Brown (SM'06) currently holds the Chair in Communication Engineering at the University of Manchester, Manchester, U.K. He joined academia in 2003 as Communications Group Leader in the School of Electrical and Electronic Engineering, University of Manchester, after a 30 year industrial career including senior board level positions. A Physicist and Mathematician by training, he has worked for GEC-Marconi Research (now BAE Systems ATC),
Standard Telecommunications Laboratories (now Nortel), University of Surrey (as the first Racal Telecommunications Fellow) and Racal before forming Easat Antennas Ltd., in 1987. Easat is recognized today as an international supplier of specialist high performance radar and communications equipment. Currently, he is retained as part time Chairman of the company.

Prof Brown is a Fellow of the Institution of Engineering and Technology (IET) and the IMA and is a Charted Engineer and Mathematician. He has served on numerous international committees including currently being a member of the Technical Advisory Commission (TAC) to the Federal Communication Commission (USA). He has had a career long involvement with computer based analysis techniques and was the first chairman of the U.K. Chapter of the Applied Computational Electromagnetics Society (1986). He has served on many national and international committees (including the IEEE and IET, EUROCAE, and ARINC). 